Research Article

\title{
A Novel Fault Diagnosis Method for Motor Bearing Based on DTCWT and AFSO-KELM
}

\author{
Yan Lu (iD) and Peijiang Li \\ Department of Information Engineering, Quzhou College of Technology, Quzhou 324000, China \\ Correspondence should be addressed to Yan Lu; luyan@qzct.net
}

Received 12 May 2021; Revised 29 May 2021; Accepted 8 June 2021; Published 17 June 2021

Academic Editor: Chaoqun Duan

Copyright (c) 2021 Yan Lu and Peijiang Li. This is an open access article distributed under the Creative Commons Attribution License, which permits unrestricted use, distribution, and reproduction in any medium, provided the original work is properly cited.

\begin{abstract}
Aiming at the defects of wavelet transform-based feature extraction and extreme learning machine-based classification, a novel fault diagnosis method for motor bearing, based on dual tree complex wavelet transform and artificial fish swarm optimizationkernel extreme learning machine (DTCWT-AFSO-KELM), is proposed in this paper. Firstly, the dual tree complex wavelet transform instead of the discrete wavelet transform is used to decompose the motor bearing signal; then, the features with large differentiation of motor-bearing fault are extracted; finally, the states of motor bearing are classified by using artificial fish swarm optimization-kernel extreme learning machine. In order to better prove the superiority of this method, four kinds of state data of motor bearing under the conditions of $0 \mathrm{HP}$ (horsepower) load, $1 \mathrm{HP}$ load, $2 \mathrm{HP}$ load, and $3 \mathrm{HP}$ load are used to test. The experimental results indicate that the diagnosis accuracies of DTCWT-AFSO-KELM are obviously better than those of discrete wavelet transform and artificial fish swarm optimization-kernel extreme learning machine (DWT-AFSO-KELM) or discrete wavelet transform and extreme learning machine (DWT-ELM) under different loads.
\end{abstract}

\section{Introduction}

Fault diagnosis of motor bearing is very significant to ensure the normal operation of the motor. At present, fault diagnosis methods of motor bearing include artificial neural network, support vector machine, and extreme learning machine. [1-3], among which extreme learning machine [4-9] has a wide application in classification and prediction fields due to its advantages of fast network training speed and good generalization performance, and extreme learning machine is a promising fault diagnosis method of motor bearing. However, it is necessary to set the number of hidden layer nodes in the training process of extreme learning machine. The existence of multicollinearity in data samples may lead to singularity, resulting in inconsistent input weights of the hidden layer, which affects the generalization performance of extreme learning machine. In this paper, kernel mapping is used to replace the random mapping of extreme learning machine, and the extreme learning machine is denoted as kernel extreme learning machine (KELM).
The penalty factor and kernel parameter of the kernel extreme learning machine need to be optimized due to the influence of the penalty factor and kernel parameter on the classification performance of the kernel extreme learning machine. Because of the shortcomings of ant colony algorithm and bee colony algorithm, artificial fish swarm optimization (AFSO) algorithm is used to optimize the parameters of kernel extreme learning machine to realize the parameter optimization of kernel extreme learning machine effectively. AFSO algorithm has the characteristics of fast search speed, high precision, and avoiding local minimum. Therefore, the kernel extreme learning machine optimized by artificial fish swarm optimization algorithm has better classification performance than ordinary extreme learning machine.

Feature extraction plays an important role in fault diagnosis of motor bearing, and signal decomposition algorithm is the key to accurately extract the running features of motor bearing. The dual tree complex wavelet transform (DTCWT) is a new wavelet transform method with many 
excellent characteristics developed in recent years. It has the advantages of approximate translation invariance, antiband aliasing, and complete reconstruction. Therefore, this paper uses dual tree complex wavelet transform to replace the commonly used discrete wavelet transform (DWT) [10-12] to decompose the motor bearing signal, which is helpful to improve the feature differentiation of different states of motor bearing in fault diagnosis of motor bearing.

Aiming at the defects of wavelet transform-based feature extraction and extreme learning machine-based classification, a novel fault diagnosis method for motor bearing based on dual tree complex wavelet transform and artificial fish swarm optimization-kernel extreme learning machine is proposed in this paper. Firstly, the dual tree complex wavelet transform is used to decompose the signal of motor bearing, and the features of large differentiation of different states of motor bearing are extracted, and the kernel extreme learning machine optimized by artificial fish swarm optimization is used to classify the state of motor bearing. The motor bearing data set of Case Western Reserve University is used as the experimental data. The common faults of motor bearing include inner ring fault, outer ring fault, and ball fault. In order to better prove the superiority of this method, four kinds of state data of motor bearing under the conditions of 0 HP load, 1 HP load, 2 HP load, and 3 HP load are used to test.

Firstly, the dual tree complex wavelet transform is introduced; secondly, the kernel extreme learning machine optimized by artificial fish swarm optimization is described; thirdly, the detailed experimental results and analysis are described; finally, conclusions are described.

\section{Dual Tree Complex Wavelet Transform}

Dual tree complex wavelet transform is a new wavelet transform method developed in recent years with many excellent characteristics, such as approximate translation invariance, antialiasing, and complete reconstruction. DTCWT adopts two parallel DWTs with different low-pass and high-pass filters. In order to obtain better symmetry, the filter length of one branch tree is odd and the other branch tree is even. There is a delay of one sampling interval between two branch tree filters, and the real part tree is missing. The sample value can be acquired by the imaginary part tree without losing the hidden information contained in the original signal hide information.

The reconstruction algorithm of dual tree complex wavelet coefficients is described as [13-15]

$$
\begin{aligned}
& d_{i}(t)=2^{(i-1) / 2}\left[\sum_{m} d_{i}^{\mathrm{Re}}(k) \phi_{h}\left(2^{i} t-m\right)+\sum_{n} d_{i}^{\mathrm{Im}}(k) \phi_{g}\left(2^{i} t-n\right)\right], \\
& c_{j}(t)=2^{(j-1) / 2}\left[\sum_{m} c_{j}^{\mathrm{Re}}(k) \varphi_{h}\left(2^{j} t-m\right)+\sum_{n} d_{i}^{\mathrm{Im}}(k) \varphi_{g}\left(2^{i} t-n\right)\right],
\end{aligned}
$$

where $m$ and $n$ denote the lengths of the real and imaginary part filters of the dual tree.
This paper uses the dual tree complex wavelet transform to replace the commonly used discrete wavelet transform to decompose the motor-bearing signal, which is helpful to improve the feature differentiation of fault diagnosis of motor bearing. The number of subsignals of the motor-bearing signal is determined according to the amplitude-frequency contrast algorithm for the purpose of achieving the separation of signal frequencies accurately.

\section{Kernel Extreme Learning Machine Optimized by Artificial Fish Swarm Optimization}

3.1. Kernel Extreme Learning Machine. The classification function of extreme learning machine is described as

$$
f(x)=h(x) \beta,
$$

where $h(x)$ is the feature mapping function matrix and $\beta=$ $\left[\beta_{1}, \ldots, \beta_{L}\right]^{T}$ is the weight vector connecting the hidden layer and the output layer.

The training objective of extreme learning machine is to calculate the output weight vector $\beta, \beta=H^{-1} T$, where $H=$ $\left[h\left(x_{1}\right), \ldots, h\left(x_{N}\right)\right]^{T}$ is the hidden layer feature mapping matrix and the training objective matrix.

For KELM, $(I / C)$ is added to the main diagonal of the unit diagonal matrix, and the KELM weight matrix is described as

$$
\beta=H^{T}\left(\frac{I}{C}+H H^{T}\right)^{-1} T,
$$

where $C$ is the penalty factor and $I$ is the identity matrix.

KELM introduces the kernel function instead of the characteristic matrix, and the corresponding KELM classification function is

$$
f(x)=\left[\begin{array}{c}
K\left(x, x_{1}\right) \\
K\left(x, x_{2}\right) \\
\cdots \\
K\left(x, x_{N}\right)
\end{array}\right]^{T}\left(\frac{I}{C}+\Delta\right)^{-1} T^{T},
$$

where $\Delta=K\left(x_{i}, x_{j}\right)$ is the kernel function, KELM selects the Gaussian radial basis function, and $K\left(x_{i}, x_{j}\right)=$ $\exp \left(-\alpha\left\|x_{i}-x_{j}\right\|^{2}\right)$, where $\alpha$ is the kernel parameter.

The penalty factor $C$ and radial basis function kernel parameter $\alpha$ of KELM need to be optimized. The suitable intelligent optimization algorithm needs to be selected to optimize the penalty factor $C$ and radial basis function kernel parameter $\alpha$ of KELM.

\subsection{Parameter Optimization of Kernel Extreme Learning} Machine Based on Artificial Fish Swarm Optimization Algorithm. Artificial fish swarm optimization algorithm makes up for the shortcomings of ant colony algorithm and bee colony algorithm [16] and has the 


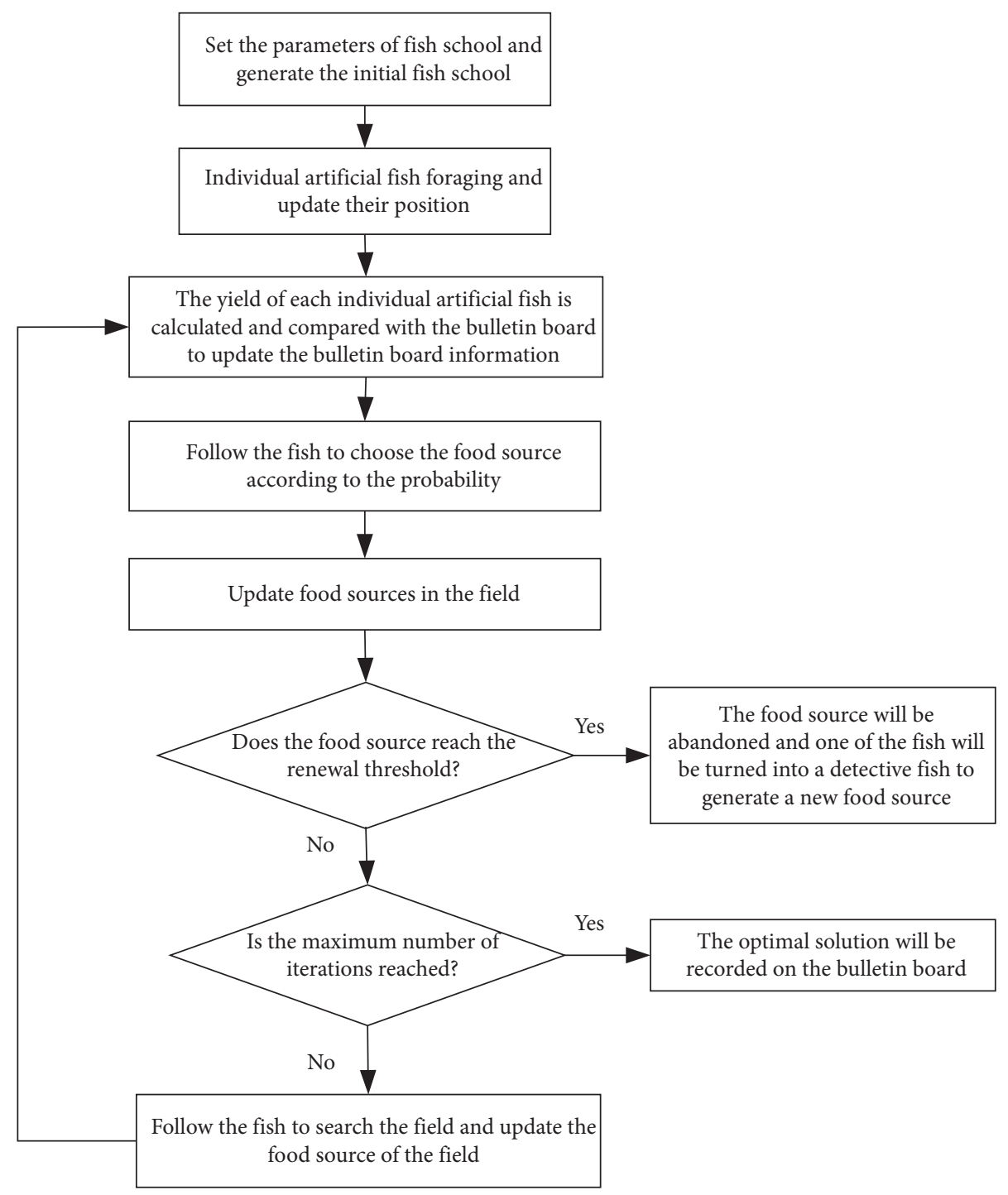

FIGURE 1: Parameter optimization of kernel extreme learning machine based on artificial fish swarm optimization algorithm.

characteristics of fast search speed, high precision, and avoiding local minimum. Therefore, the parameters of kernel extreme learning machine can be effectively optimized by artificial fish swarm optimization algorithm. The process of optimizing kernel extreme learning machine by artificial fish swarm is shown in Figure 1, and the optimization process of kernel extreme learning machine by artificial fish swarm optimization is described as follows:

Step 1: KELM's penalty factor $C$ and radial basis function kernel parameter $\alpha$ constitute the individual position of the artificial fish, and the parameters of the fish group are set, including the size of the fish group $m$, the maximum number of iterations, and the moving step; $m$ artificial fish individuals are randomly generated as the initial fish group;

Step 2: individual artificial fish foraging and update their position according to the following formula:

$$
Y_{\text {inext }}=Y_{p}+\left(\text { step } \cdot \frac{Y_{c}-Y_{i}}{\left\|Y_{c}-Y_{i}\right\|}\right)+\left(\operatorname{step} \cdot \frac{Y_{\max }-Y_{i}}{\left\|Y_{c}-Y_{i}\right\|}\right)
$$

where $Y_{c}$ is the center position of the whole artificial fish group, $Y_{\max }$ is the optimal position of the whole artificial fish group at present, $Y_{p}$ is the position of the artificial fish after foraging behavior, and $Y_{i}$ is the current position of the artificial fish.

Step 3: the yield of each individual artificial fish is calculated and compared with the bulletin board to update the bulletin board information.

Step 4: follow the fish to choose the food source according to the probability.

Step 5: update food sources in the field.

Step 6: check the update threshold of the food source. If the update threshold is reached, the food source will be abandoned and one of the fish will be turned into a 


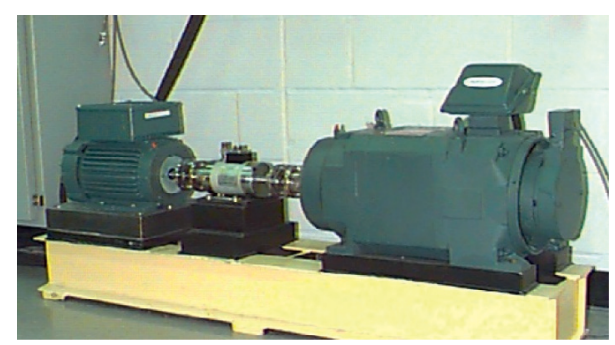

Figure 2: The experimental device.

detective fish to generate a new food source; otherwise, go to step 7.

Step 7: check whether the maximum number of iterations is satisfied. If it is satisfied, the optimal solution will be recorded on the bulletin board, namely, the penalty factor $C$ and the radial basis function kernel parameter $\alpha$ of the optimal KELM are output, and the algorithm ends; otherwise, calculate the fish yield, update the bulletin board information, and go to step 3 .

\section{Experimental Results and Analysis}

The motor bearing data set of Case Western Reserve University is used as the experimental data. The experimental device is shown in Figure 2 [17]. Motor-bearing faults are usually inner ring fault, outer ring fault, and ball fault. In order to better prove the superiority of this proposed method, four kinds of state data of motor bearing under 0 HP load, 1 HP load, 2 HP load, and 3 HP load are used to test. Firstly, 280 samples (70 samples expressing normal state, 70 samples expressing inner ring fault, 70 samples expressing outer ring fault, and 70 samples expressing ball fault) of motor bearing under $0 \mathrm{HP}$ load were used as the training samples and another 200 samples (50 samples expressing normal state, 50 samples expressing inner ring fault, 50 samples expressing outer ring fault, and 50 samples expressing ball fault) of motor bearing under $0 \mathrm{HP}$ load were tested. Secondly, 280 samples (70 samples expressing normal state, 70 samples expressing inner ring fault, 70 samples expressing outer ring fault, and 70 samples expressing ball fault) of motor bearing under $1 \mathrm{HP}$ load were used as the training samples and another 200 samples (50 samples expressing normal state, 50 samples expressing inner ring fault, 50 samples expressing outer ring fault, and 50 samples expressing ball fault) of motor bearing under $1 \mathrm{HP}$ load were tested. Thirdly, 280 samples (70 samples expressing normal state, 70 samples expressing inner ring fault, 70 samples expressing outer ring fault, and 70 samples expressing ball fault) of motor bearing under $2 \mathrm{HP}$ load were used as the training samples and another 200 samples (50 samples expressing normal state, 50 samples expressing inner ring fault, 50 samples expressing outer ring fault, and 50 samples expressing ball fault) of motor bearing under $2 \mathrm{HP}$ load were tested. Finally, 280 samples (70 samples expressing normal state, 70 samples expressing inner ring fault, 70 samples expressing outer ring fault, and 70 samples expressing ball fault) of motor bearing under $3 \mathrm{HP}$ load were used as the
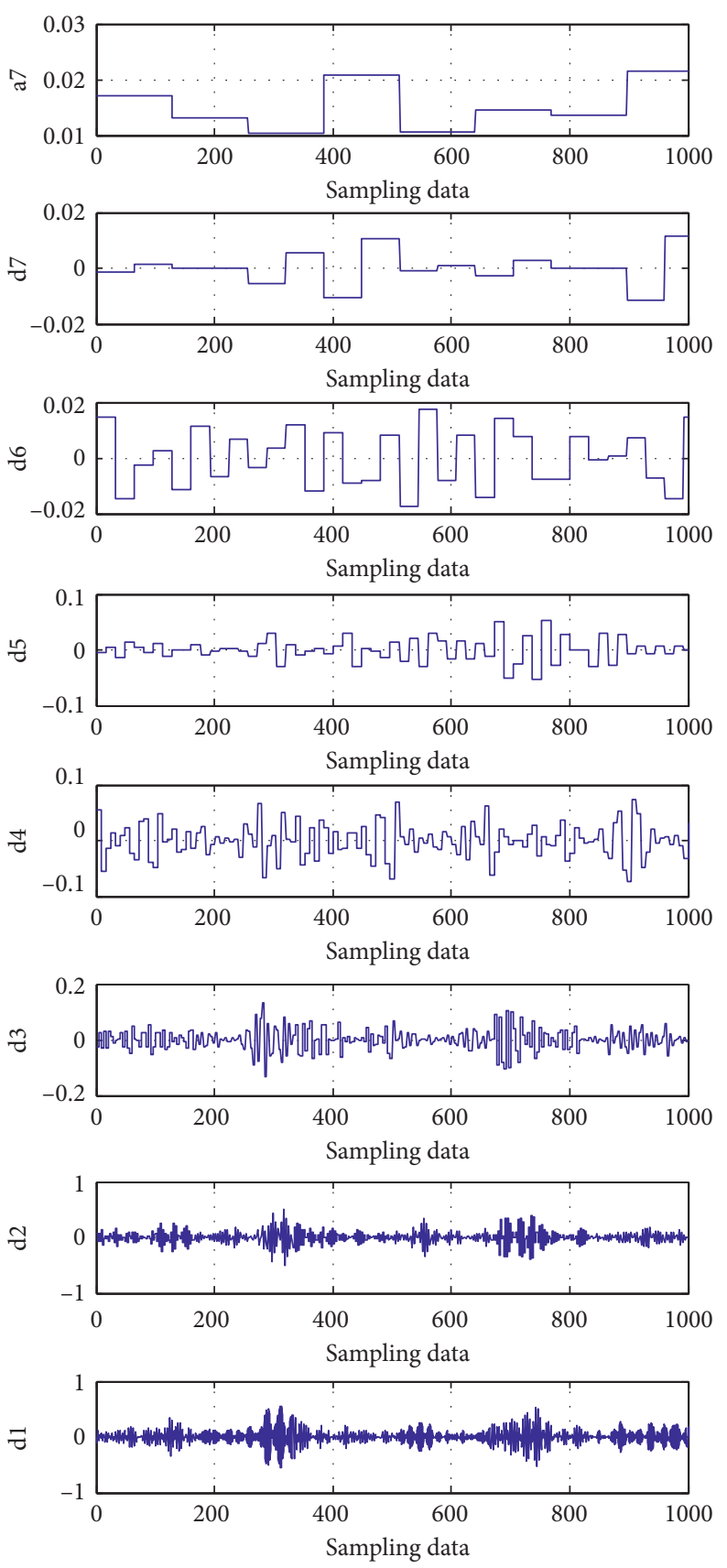

FIgURE 3: The decomposition signal of motor bearing based on the dual tree complex wavelet transform.

training samples and another 200 samples (50 samples expressing normal state, 50 samples expressing inner ring fault, 50 samples expressing outer ring fault, and 50 samples expressing ball fault) of motor bearing under $3 \mathrm{HP}$ load were tested. The decomposition signal of motor bearing based on the dual tree complex wavelet transform is shown in Figure 3.

Figure 4 shows the diagnosis results of DTCWT-AFSOKELM, DWT-AFSO-KELM, and DWT-ELM under different load conditions. As shown in Table 1, under 0 HP load condition, the diagnosis accuracy of motor bearing by using DTCWT-AFSO-KELM is $99.5 \%$, the diagnosis accuracy of 


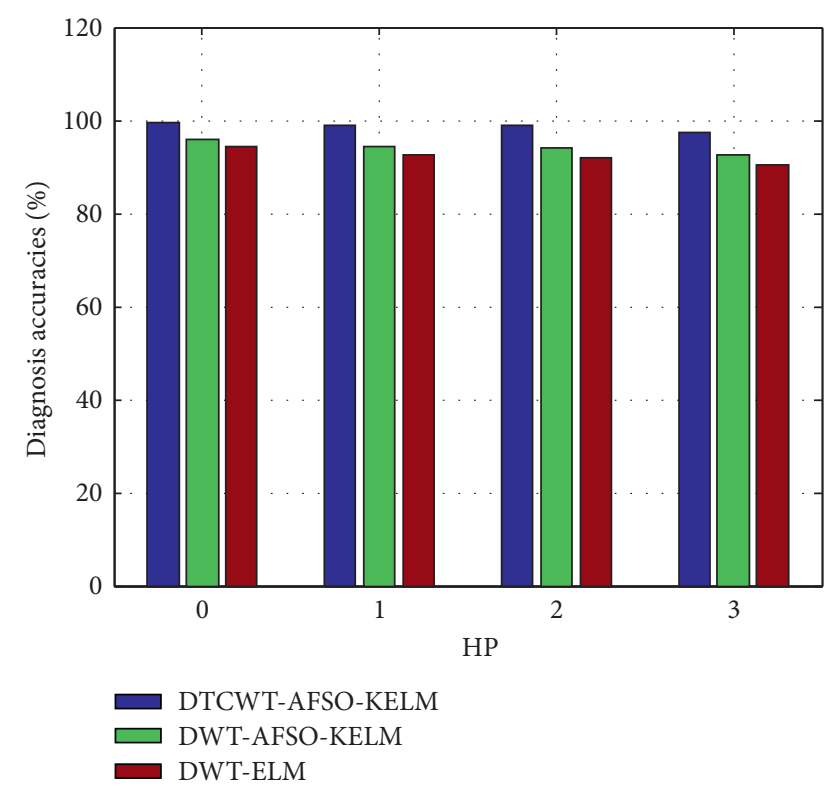

FIGURE 4: The diagnosis results of DTCWT-AFSO-KELM, DWTAFSO-KELM, and DWT-ELM under different load conditions.

TABle 1: The diagnosis accuracies of DTCWT-AFSO-KELM, DWT-AFSO-KELM, and DWT-ELM under different load conditions.

\begin{tabular}{lcc}
\hline Load & Diagnosis method & Diagnosis accuracy (\%) \\
\hline \multirow{3}{*}{0 HP } & DTCWT-AFSO-KELM & 99.5 \\
& DWT-AFSO-KELM & 96 \\
& DWT-ELM & 94.5 \\
\hline \multirow{3}{*}{$1 \mathrm{HP}$} & DTCWT-AFSO-KELM & 99 \\
& DWT-AFSO-KELM & 94.5 \\
& DWT-ELM & 92.5 \\
\hline \multirow{3}{*}{$2 \mathrm{HP}$} & DTCWT-AFSO-KELM & 99 \\
& DWT-AFSO-KELM & 94 \\
& DWT-ELM & 92 \\
\multirow{3}{*}{$3 \mathrm{HP}$} & DTCWT-AFSO-KELM & 97.5 \\
& DWT-AFSO-KELM & 92.5 \\
& DWT-ELM & 90.5 \\
\hline
\end{tabular}

motor bearing by using DWT-AFSO-KELM is $96 \%$, and the diagnosis accuracy of motor bearing by using DWT-ELM is $94.5 \%$. Under $1 \mathrm{HP}$ load condition, the diagnosis accuracy of motor bearing by using DTCWT-AFSO-KELM is $99 \%$, the diagnosis accuracy of motor bearing by using DWT-AFSOKELM is $94.5 \%$, and the diagnosis accuracy of motor bearing by using DWT-ELM is $92.5 \%$. Under $2 \mathrm{HP}$ load condition, the diagnosis accuracy of motor bearing by using DTCWTAFSO-KELM is 99\%, the diagnosis accuracy of motor bearing by using DWT-AFSO-KELM is $94 \%$, and the diagnosis accuracy of motor bearing by using DWT-ELM is $92 \%$. Under 3 HP load condition, the diagnosis accuracy of motor bearing by using DTCWT-AFSO-KELM is $97.5 \%$, the diagnosis accuracy of motor bearing by using DWT-AFSOKELM is $92.5 \%$, and the diagnosis accuracy of motor bearing by using DWT-ELM is $90.5 \%$. It can be seen that the diagnosis results of motor bearing by using DTCWT-AFSO-
KELM are obviously better than those by using DWTAFSO-KELM or DWT-ELM.

\section{Conclusions}

In this paper, a novel fault diagnosis method for motor bearing based on dual tree complex wavelet transform and artificial fish swarm optimization-kernel extreme learning machine is proposed because of the defects of wavelet transform-based feature extraction and extreme learning machine-based classification. The contributions of this paper are reduced as follows:

(1) The dual tree complex wavelet transform is used to replace the commonly used discrete wavelet transform to decompose the motor bearing signal, which is helpful to improve the feature differentiation of different states of motor bearing in fault diagnosis of motor bearing.

(2) The parameters of kernel extreme learning machine are optimized by artificial fish swarm optimization algorithm which has the characteristics of fast search speed, high precision, and avoiding local minimum, and the artificial fish swarm optimization-kernel extreme learning machine is used to classify the state of motor bearing. The experimental results show that the diagnosis accuracies of DTCWT-AFSO-KELM are obviously better than those of DWT-AFSOKELM or DWT-ELM. The future research and development focus on the improvement for artificial fish swarm optimization algorithm to obtain the kernel extreme learning machine with more excellent classification performance.

\section{Data Availability}

The data that support the findings of the research are available from the corresponding author.

\section{Conflicts of Interest}

The authors declare no conflicts of interest.

\section{Acknowledgments}

This work was supported by the Basic Public Welfare Research Project of Zhejiang Province (LGG18E050003) and the Second Batch of Teaching Reform Research Projects in the 13th Five-Year Plan of Zhejiang Province (jg20190891).

\section{References}

[1] D.-T. Hoang and H.-J. Kang, "Rolling element bearing fault diagnosis using convolutional neural network and vibration image," Cognitive Systems Research, vol. 53, pp. 42-50, 2019.

[2] X. Yan and M. Jia, "A novel optimized svm classification algorithm with multi-domain feature and its application to fault diagnosis of rolling bearing," Neurocomputing, vol. 313, pp. 47-64, 2018.

[3] J. Zheng, Z. Dong, H. Pan, Q. Ni, T. Liu, and J. Zhang, "Composite multi-scale weighted permutation entropy and 
extreme learning machine based intelligent fault diagnosis for rolling bearing," Measurement, vol. 143, pp. 69-80, 2019.

[4] Y. Li, Y. Zeng, Y. Qing, and G.-B. Huang, "Learning local discriminative representations via extreme learning machine for machine fault diagnosis," Neurocomputing, vol. 409, pp. 275-285, 2020.

[5] Y. Qing, Y. Zeng, Y. Li, and G.-B. Huang, "Deep and wide feature based extreme learning machine for image classification," Neurocomputing, vol. 412, pp. 426-436, 2020.

[6] S. Shukla and B. S. Raghuwanshi, "Online sequential classspecific extreme learning machine for binary imbalanced learning," Neural Networks, vol. 119, pp. 235-248, 2019.

[7] F. Olivetti de Franca and M. Zabuscha de Lima, "Interactiontransformation symbolic regression with extreme learning machine," Neurocomputing, vol. 423, pp. 609-619, 2020.

[8] G. S. Nandini and A. P. S. Kumar, "Dropout technique for image classification based on extreme learning machine," Global Transitions Proceedings, vol. 2, no. 1, pp. 111-116, 2021.

[9] A. Law and A. Ghosh, "Multi-label classification using a cascade of stacked autoencoder and extreme learning machines," Neurocomputing, vol. 358, pp. 222-234, 2019.

[10] R. Kamgar, M. Dadkhah, and H. Naderpour, "Seismic response evaluation of structures using discrete wavelet transform through linear analysis," Structure, vol. 29, pp. 863-882, 2021.

[11] K. Gopala Krishnan and P. T. Vanathi, "An efficient texture classification algorithm using integrated discrete wavelet transform and local binary pattern features," Cognitive Systems Research, vol. 52, pp. 267-274, 2018.

[12] J. Nobre and R. F. Neves, "Combining principal component analysis, discrete wavelet transform and xgboost to trade in the financial markets," Expert Systems with Applications, vol. 125, pp. 181-194, 2019.

[13] N. Aishwarya and C. Bennila Thangammal, "Visible and infrared image fusion using dtcwt and adaptive combined clustered dictionary," Infrared Physics \& Technology, vol. 93, pp. 300-309, 2018.

[14] Z. He, Z. Tang, Z. Yan, and J. Liu, "DTCWT-based zinc fast roughing working condition identification," Chinese Journal of Chemical Engineering, vol. 26, no. 8, pp. 1721-1726, 2018.

[15] I. J. Kadhim, P. Premaratne, and P. J. Vial, "High capacity adaptive image steganography with cover region selection using dual-tree complex wavelet transform," Cognitive Systems Research, vol. 60, pp. 20-32, 2020.

[16] K. P. Kumar, B. Saravanan, and K. S. Swarup, "Optimization of renewable energy sources in a microgrid using artificial fish swarm algorithm," Energy Procedia, vol. 90, pp. 107-113, 2016.

[17] Case Western Reserve University, Bearing Data Center[DB/ $O L]$, Case Western Reserve University, Cleveland, OH, USA, 2017, https://csegroups.case.edu/bearingdatacenter. 